\title{
A NOTE ON TWO GENERATING FUNCTIONS FOR LEGENDRE FUNCTIONS
}

NICHOLAS D. KAZARINOFF

It has recently been shown by Bloch [1] that for Legendre functions not on the cut

$$
e^{t z} I_{m}\left[t\left(z^{2}-1\right)^{1 / 2}\right]=\sum_{n=0}^{\infty} P_{m+n}^{m}(z) \frac{t^{m+n}}{(2 m+n) !}, \quad m=0,1,2, \cdots,
$$

where

$$
I_{m}(z)=\sum_{n=0}^{\infty} \frac{(z / 2)^{m+2 n}}{n !(m+n) !}, \quad P_{n}^{m}(z)=\left(z^{2}-1\right)^{m / 2} d^{m} P_{n}(z) / d z^{m} .
$$

It is the purpose of this note to show how (1) and the result (2) below may be derived using methods of Truesdell [2] and thus to correct the results (58) and (66) on pages 101 and 105 of [2].1

If one applies Theorem 14.4 of [2] to the function

$$
F(z, \alpha)=\frac{\cos \pi(\alpha-m)}{\Gamma(1-\alpha+2 m)}\left(z^{2}-1\right)^{-(\alpha-m) / 2} P_{m-\alpha}^{m}\left[\frac{-z}{\left(z^{2}-1\right)^{1 / 2}}\right],
$$

the changes of variable $x=-z\left(z^{2}-1\right)^{-1 / 2}, y z=x t$ lead after simplifications to the generating function relationship (1). Application of Theorem 14.7 of [2] to the function

$$
F(z, \alpha)=\Gamma(\alpha-b+1)\left(z^{2}-1\right)^{-(\alpha+1) / 2} P_{\alpha}^{b}\left[\frac{-z}{\left(z^{2}-1\right)^{1 / 2}}\right]
$$

gives the formula

$$
\begin{gathered}
\sum_{n=0}^{\infty} \frac{\Gamma(\alpha+n-b+1)}{n ! \Gamma(\alpha+n+1)}\left(z^{2}-1\right)^{-(\alpha+n+1) / 2} P_{\alpha+n}^{b}\left[\frac{-z}{\left(z^{2}-1\right)^{1 / 2}}\right] y^{n} \\
=\frac{\Gamma(\alpha-b+1)}{2 \pi i} \int_{c} e^{w} w^{-\alpha}\left[(z+y / w)^{2}-1\right]^{-(\alpha+1) / 2} \\
\cdot P_{\alpha}^{b}\left[\frac{-z-y / w}{\left\{(z+y / w)^{2}-1\right\}^{1 / 2}}\right] d w
\end{gathered}
$$

Received by the editors May 16, 1955 .

1 Truesdell's results are incorrect because of computational error. The incorrect formula 19.10(17) of [3] should also be replaced by (1). 
where the contour of integration extends from $-\infty$ counterclockwise about the points $y /(1-z)$ and $-y /(1+z)$ and back to $-\infty$. Upon making the substitutions $\alpha=b=m, m=0,1,2, \cdots, x=-z\left(z^{2}-1\right)^{-1 / 2}$, $y\left(z^{2}-1\right)^{-1 / 2}=t$, and $w=t s$, one finds after simplifications that for the Legendre functions away from the cut

$$
\begin{aligned}
& \frac{(2 m-1) ! !\left(x^{2}-1\right)^{m / 2}}{2 \pi i} \int_{C} e^{s t}\left(1-2 x s+s^{2}\right)^{-m-1 / 2} s^{m} d s \\
& =\sum_{n=0}^{\infty} P_{n+m}^{m}(x) \frac{t^{m+n}}{(m+n) !} .
\end{aligned}
$$

The variables $y$ and $t$ are real. The contour $C$ now extends from $-\infty$ counterclockwise around the zeros of $\left(1-2 x s+s^{2}\right)$ and back to $-\infty$.

\section{REFERENCES}

1. E. L. Bloch, On an expansion of Bessel functions in a series of Legendre functions, Akad. Nauk SSSR. Prikl. Mat. Meh. vol. 18 (1954) pp. 745-748.

2. C. Truesdell, $A$ unified theory of special functions, Princeton University Press, 1948.

3. The Bateman Manuscript Project, Higher transcendental functions, vol. 3, McGraw-Hill, 1955.

Purdue University 